\title{
Knowledge regarding Zika disease among healthcare professionals of tricity: A cross-sectional questionnaire study
}

\author{
Amandeep Chopra \\ Senior Lecturer, Dept. of Public Health Dentistry, National Dental College and Hospital, Derabassi, Punjab, India
}

Corresponding Author:

Email: dr.amandeepchopra@gmail.com

\begin{abstract}
Introduction: Zika virus is prompting worldwide concern because of an alarming connection to a neurological birth disorder and the rapid spread of the virus across the globe. Health care professionals who come in contact with patients should be aware of this disease. The purpose of this study was to assess the knowledge of Healthcare professionals towards Zika Virus in Tricity, India.

Materials and Method: A total of 510 healthcare practitioners were randomly approached to participate in this cross-sectional survey. A self-structured, closed ended questionnaire was administered to each participant to record demographic and professional characteristics followed by their knowledge regarding Zika virus. Multivariable linear regression analysis was carried out to assess the association of participant's demographic and professional characteristics with the knowledge scores.

Result: The mean knowledge score of the respondents was $12.57 \pm 0.42$. Multivariate regression analysis revealed that age, location, profession and years of clinical practice had a significant impact on knowledge score.

Conclusion: Healthcare professionals from urban areas, medical profession and with higher clinical practice had better knowledge yet there were notable deficiencies regarding the virus characteristics, diagnostics, elimination and treatment.
\end{abstract}

Keywords: Zika virus, Knowledge, Prevention, Healthcare professionals.

\section{Introduction}

Zika virus, a mosquito based virus, has caught attention worldwide as being associated with neurological birth disorder and is spreading at a striking rate. ${ }^{1,2}$ Zika is a flavivirus transmitted by Aedes mosquito which was first isolated in 1947 in Rhesus macaques of the Zika Forest (Uganda); human infection was initially shown in 1952 and the virus was successfully isolated from human samples in Nigeria in $1968 .^{3}$

Symptoms associated with Zika virus infection are mild fever and exanthema (skin rash), sometimes accompanied by conjunctivitis, muscle or joint pain, and general malaise manifesting 2-7 days post infected mosquito bite. These symptoms are comparable to other mosquito borne diseases such as Dengue and Chikungunya. ${ }^{4}$ Apart from bite of Aedes mosquito being the primary etiological factor (A. aegypti and A. albopictus) it can be transmitted rarely from mother to child and through infected blood or sexual contact. ${ }^{5}$ The clusters of microcephaly and Guillain-Barré Syndrome (GBS) that have been temporally associated with Zikavirus transmission in some settings (such as Brazil) has led Director-General of World Health Organization to declare Public Health Emergency of International Concern (PHEIC) on 1 February 2016. ${ }^{6,7}$

Zika virus is gradually spreading as a pandemic due to well connected globe across and ease of travel from one place to another, hence health professionals having thorough knowledge of Zika virus and its contagious nature is becoming indispensable. Many parts of India have poor mosquito control, these areas remain vulnerable if an infected individual reaches those regions and gets exposed to mosquitoes. Healthcare professionals are required to have a knowledge about mode of transmission, pathogenesis, clinical symptoms and prompt treatment measures related to Zika virus. The basic objective of this study was to assess the knowledge of Healthcare professionals towards Zika Virus in Tricity, India.

\section{Materials and Method}

A descriptive cross-sectional questionnaire study was conducted from December 2015 to January 2016. Ethical clearance was obtained from the Institutional Ethical Committee and oral informed consent was obtained from the participants. A self-structured, closed ended questionnaire was provided to each participant in person, which took approximately comprising of 20 minutes details. Anonymity and confidentiality of each patient was ensured. A pilot test of original version of questionnaire was done in order to determine the testretest reliability of the survey questions, twenty healthcare practitioners (7 Medical, 7 Dental and 6 Nursing) were included in the survey and duration was two weeks. Feedback was also taken from the participants regarding the clarity of questions or any ambiguity associated in answering them. The participants of the pilot study were kept excluded from the final test sample. Chronbach's alpha of the questionnaire was found to be acceptable (0.84). Few modifications were incorporated to improve the understanding of the questionnaire based on the responses. Based on pilot study sample size was estimated for the main study $(\mathrm{n}=510)$. List of healthcare practitioners (Medical, Nursing and Dental) was obtained from the respective registration council 
branches across Chandigarh, Panchkula and Mohali. Then 170 members each in Medical, Dental and Nursing were selected by simple random sampling. Participants who were not willing to participate in the study or who were absent during three consecutive visits were excluded from the study.

Final questionnaire consisted of 3 sections: a. demographic and professional details (age, gender, qualification, and years of practice); b. knowledge of Zika disease (20questions) included questions regarding communicability; symptomatology and diagnostics; atrisk individuals; prevention and treatment; virus characteristics; and source of knowledge regarding Zika disease. The question designing was done after an intensive literature review by the research team. The responses to these questions were of multiple-choice type having one correct answer, including an option of "don't know" in some of questions.

The data was compiled and entered into Standard Microsoft Excel 2013. Statistical analysis was conducted using SPSS computer package version 20.0 (SPSS Inc., Chicago, IL, USA).The results were expressed in percentages. To assess the association of participants demographic knowledge, multivariable linear regression analysis was carried out with significance level of $\mathrm{p}<0.05$ was set throughout.

\section{Result}

510 healthcare professionals in total were approached to participate in the survey, out of which 486 gave their free will to complete the questionnaire. $5 \%(24 / 486)$ of healthcare professionals don't know about Zika disease. Table 1 presents the sociodemographic characteristic of the population.

The respondents had a mean (also the median) knowledge score of $2.57 \pm 0.42$ (Table 2). The responses to the knowledge questions are detailed in Table3.

Multivariable linear regression analysis was carried out to find out the determinants of knowledge among the study participants. Of the five demographic and professional characteristics (age, location, gender) profession and years of clinical practice) were included into the model, Gender variation regarding knowledge score was found to be non significant. Other factors such as age, location, profession and years of clinical practice had a significant impact on knowledge score (Table 4).

Table 5 represents the origin of information about Zika among the study participants.

Table 1: Demographic and professional characteristics of the participants of the study

\begin{tabular}{|l|c|c|}
\hline Characteristics & Frequency & Percentage \\
\hline Gender & & \\
\hline Male & 281 & 57.8 \\
\hline Female & 205 & 42.2 \\
\hline Age (years) & & \\
\hline
\end{tabular}

J Dent Specialities.2018;6(1):38-42

\begin{tabular}{|l|c|c|}
\hline $25-35$ & 221 & 45.5 \\
\hline $36-45$ & 164 & 33.7 \\
\hline$>46$ & 101 & 20.8 \\
\hline Years of Practice & & \\
\hline$<5$ & 274 & 56.4 \\
\hline $5-10$ & 143 & 29.4 \\
\hline$>10$ & 69 & 14.2 \\
\hline Location & & \\
\hline Urban & 351 & 72.2 \\
\hline Rural & 135 & 28.8 \\
\hline Profession & & \\
\hline Dental & 164 & 33.7 \\
\hline Medical & 154 & 31.6 \\
\hline Nursing & 168 & 34.5 \\
\hline
\end{tabular}

Table 2: Knowledge among study participants regarding Zika

\begin{tabular}{|l|c|}
\hline Measure & Knowledge \\
\hline Mean Score & 12.57 \\
\hline Standard Error of mean & 0.42 \\
\hline Median & 12.0 \\
\hline SD & 3.34 \\
\hline $25^{\text {th }}$ Percentile & 8.51 \\
\hline $50^{\text {th }}$ Percentile & 12.0 \\
\hline $75^{\text {th }}$ Percentile & 14.20 \\
\hline
\end{tabular}

Table 3: Responses to knowledge questions regarding Zika by the participants of the study

\begin{tabular}{|l|c|}
\hline \multicolumn{1}{|c|}{ Question } & Percentage \\
\hline 1. Origin of Zika disease is in & \\
a) Brazil & 31.5 \\
b) Uganda & 44.3 \\
c) Kenya & 2.2 \\
d) Nigeria & 7.8 \\
e)Don't know & 14.2 \\
\hline 2. 1st documented case of Zika? & \\
a) 1947 & 38.0 \\
b) 1954 & 9.0 \\
c) 2005 & 2.6 \\
d) 2015 & 38.8 \\
e)Don't know & 11.6 \\
\hline 3. Isit a communicable disease? & \\
a) Yes & 69.5 \\
b) No & 15.6 \\
c) Don't know & 14.9 \\
\hline 4. Main vector for Zika? & \\
a) Birds & 2.2 \\
b) Rodents & 1.4 \\
c) Insects & 78.0 \\
d) Humans & 9.9 \\
e)Don't know & 8.5 \\
\hline 5. Pathogen causing Zika? & \\
a) Viral & 78.9 \\
b) Bacteria & 3.0 \\
c) Fungal & 1.3 \\
d) Protozoal & 9.9 \\
e)Don't know & 6.9 \\
\hline
\end{tabular}

e)Don't know 


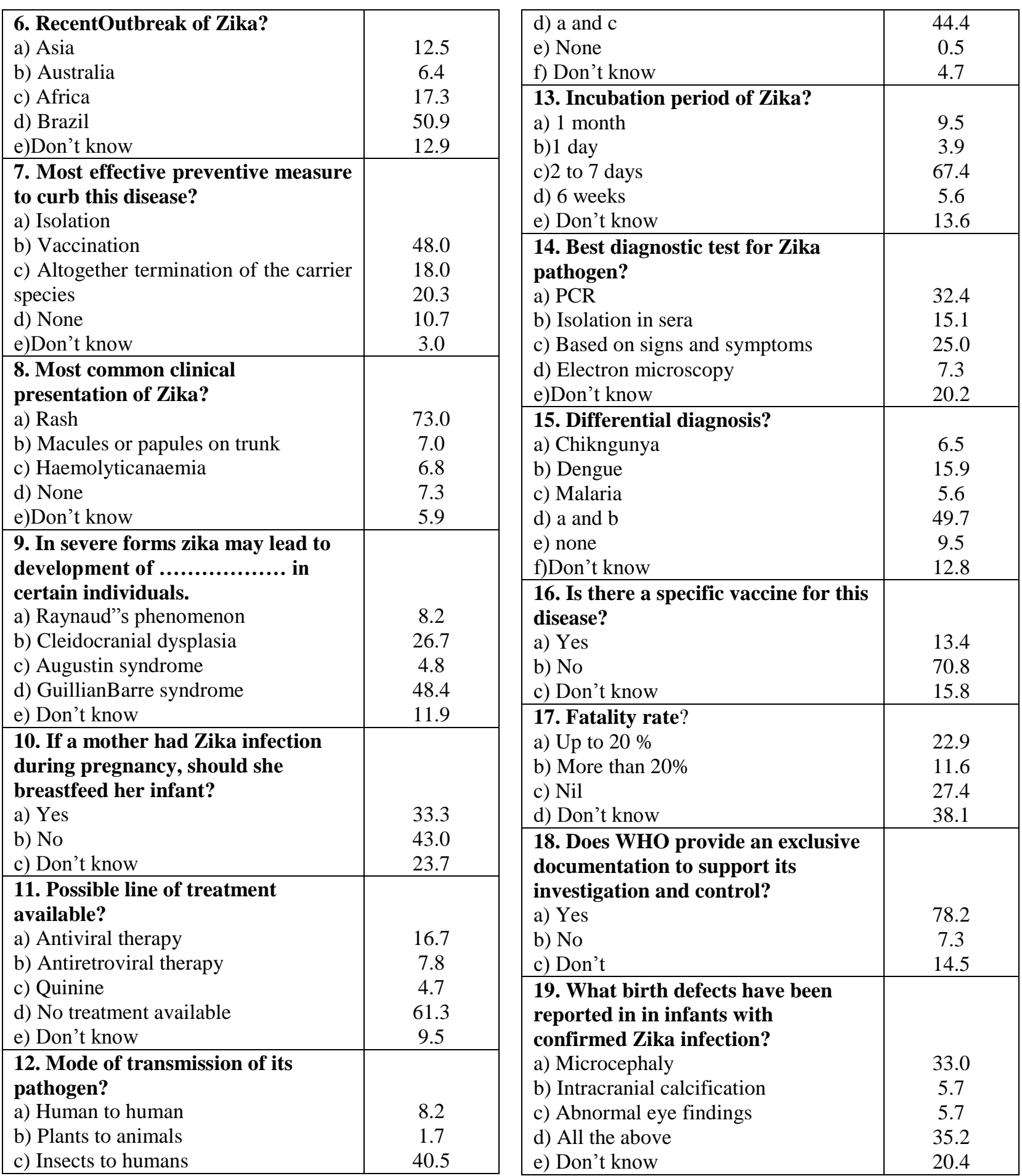


Table 4: Association between the demographic and professional characteristics of the participants with the knowledge scores using multivariate linear regression analysis

\begin{tabular}{|l|l|l|l|l|l|l|l|}
\hline \multicolumn{1}{|c|}{ Variable } & \multicolumn{1}{c|}{ B } & SE & df & Sig. & Exp. (B) & \multicolumn{2}{l|}{ 95\% CI for Exp (B) } \\
\hline Gender & & & & & & & \\
\hline Male & Referent & & & & & & \\
\hline Female & 0.470 & 0.581 & 1 & 0.413 & 0.632 & 0.210 & 1.646 \\
\hline Years of Practice & & & & & & & \\
\hline$<5$ & Referent & & 2 & 0.000 & & & \\
\hline $5-10$ & 1.641 & 0.181 & 1 & 0.000 & 5.605 & 3.820 & 8.286 \\
\hline$>10$ & 2.069 & 0.369 & 1 & 0.000 & 8.650 & 4.397 & 17.362 \\
\hline Age (years) & & & & & & & \\
\hline$>46$ & Referent & & 2 & 0.000 & & & \\
\hline $36-45$ & -1.373 & 0.273 & 1 & 0.003 & 0.217 & 0.108 & 0.426 \\
\hline $25-35$ & -1.606 & 0.336 & 1 & 0.000 & 0.172 & 0.082 & 0.348 \\
\hline Location & & & & & & & \\
\hline Urban & Referent & & & & & & \\
\hline Rural & 0.631 & 0.172 & 1 & 0.001 & 1.841 & 1.292 & 2.521 \\
\hline Profession & & & & & & & \\
\hline Nursing & Referent & & 2 & 0.000 & & & \\
\hline Dental & 1.114 & 0.312 & 1 & 0.000 & 2.856 & 1.624 & 4.993 \\
\hline Medical & 1.007 & 0.389 & 1 & 0.006 & 3.015 & 1.373 & 6.517 \\
\hline Constant & 0.117 & 0.290 & 1 & 0.630 & 1.013 & & \\
\hline
\end{tabular}

Note $=*$ Dependent variable: knowledge score regarding zika

$* * \mathrm{~B}=$ Unstandardized coefficients

$* * * \operatorname{Exp}(\mathrm{B})=$ Standardized coefficient

Table 5: Source of information about Zika among the study participants

\begin{tabular}{|l|c|}
\hline \multicolumn{1}{|c|}{ Source of information } & Percentage \\
\hline a) Print media & 13.4 \\
b) Television or radio & 11.2 \\
c) Relatives/friends/colleagues & 15.4 \\
d) Social media & 45.0 \\
e) Others & 15.0 \\
\hline
\end{tabular}

\section{Discussion}

Zika virus has increased 'exponentially' now, enough for the WHO to declare it as an international public health emergency. India has been is the in grip of its worst dengue fever outbreak in previous years which is mosquito borne disease. ${ }^{8}$ India needs to work out how it will prevent a similar outbreak of the Zika virus which is spread by same mosquitoes which spreads dengue. It is hence important that health workers be familiar with the disease manifestations, treatment and precautions to be taken while dealing with ailment as well as crossinfection. Therefore the current survey was performed to furnish the coherent description of healthcare professional's knowledge with regards to Zika.

According to Center for Disease Control and Prevention (CDC), during first 7 days of illness, viral DNA often be identified in serum, and RT-PCR is preferred diagnostic test for detection. ${ }^{9}$ It is a matter of concern that in our study participants didn't have much awareness about diagnostic test for the virus.

The results of the "knowledge level test" among the respondents to the questionnaire, (which contained questions pertaining to the communicable nature. symptoms and diagnostics; prevention and treatment; and, virus characteristics of Zika), was suggestive of the fact that knowledge about the virus among health professionals were fairly good. However, there are certain interesting yet disturbing details that have been found regarding gap in knowledge. Only 48\% participants knew that isolation needs to be done to prevent the transmission of virus to others (including pregnant women) by preventing contact between the patient infected with Zika virus and mosquitoes, at least during the first week that is viremic phase, applying the personal protection measures. ${ }^{10}$ Only $33.3 \%$ of study participants feel that if a mother had Zika infection during pregnancy, she shouldn't breastfeed her infant. Although RNA of Zika virus have been isolated from breast milk but no reports of contamination through breastfeeding has been reported as yet. Based on available facts the protective advantages that breastmilk has to offer to infants outweigh any theoretical risk associated with Zika virus infection. ${ }^{11}$

Center for Disease Control and Prevention have clearly issued guidelines for Pediatric Healthcare Providers that Zika virus is responsible for causing microcephaly, intracranial calcifications and abnormal ocular findings in infants still only $35 \%$ of study participants were aware of same.

Although WHO has provided documentation ${ }^{12,13}$ regarding Zika disease but $78.2 \%$ of the participants were aware of this, so health care professionals should 
need to regularly keep upgrading their knowledge about the virus and epidemics of such diseases that will help in inculcating positive attitude and healthy practices in them.

In the present study medical professionals data depicted that they have better knowledge than their dental and nursing counterparts, the reason for which needs to be explored. Healthcare professionals based in urban areas had significantly higher knowledge scores. This can be due to greater number of education programmes, awareness sessions and conferences conducted in urban areas in order to regularly update their knowledge about various fields including the prevention and spread of any infectious disease.

If made a gender based comparison, knowledge score was found to be same in males and females. This shows that females of this area were equally interactive and well informed as males and also they are equally concerned about increasing their knowledge banks.

Social media was found to be the most important source of information for Zika. This could be because social media tools such as Twitter and Facebook have widespread existence and reaching out directly to more health care professionals and public.

In this study merely the knowledge score has been considered thus it would be bit premature to assess the attitudes and practices in relation to Zika, when very less has been established about the disease. Nevertheless this study puts foundation of new research in this very field, as this is one of its kind studies in dental and medical literature assessing the knowledge regarding Zika disease.

\section{Conclusion}

Through this study we are in position to establish healthcare professional knowledge regarding Zika disease was fairly good. Healthcare professionals from urban areas, medical profession and with higher clinical practice possessed better knowledge yet there were not able distinguish regarding the virus characteristics, diagnostics, elimination and treatment.

Zika virus outbreaks could cause additional burdens on all levels of the health care system, it is necessary to develop and implement protocols and well established plans for the patient screening and treatment. Emphasis should be given to create a positive perception towards public health significance of diseases as well as more number of continued education programmes should be carried out to disseminate the knowledge effectively regarding Zika.

\section{References}

1. Petersena E, Wilson ME, Touch S, McCloskey B, Mwabae $\mathrm{P}$, Batese $\mathrm{M}$ et al. Rapid spread of Zika Virus in the americas - Implications for public health preparedness for mass gatherings at the 2016 Brazil Olympic Games. Int J Infect Dis 2016;44:11-4.

2. Sikka V, Chattu VK, Popli RK, Galwankar SC, Kelkar D, Sawick SG et al. The emergence of Zika Virus as a global health security threat: A review and a consensus statement of the INDUSEM Joint Working Group (JWG). J Glob Infect Dis 2016;8(1)3-15.

3. Hayes EB. Zika Virus outside Africa. Emerg Infect Dis 2009;15(9):1347-50.

4. Pan American Health Organization. Zika virus infection and Zika fever: Frequently asked questions. February 2, 2016. Available at http://www.paho.org/hq/index.php?option=com_content\&vi ew $=$ article \&id=9183\&Itemid $=41711 \&$ lang $=$ en

5. Passi D, Sharma S, Dutta SR, Ahmed M. ika Virus Diseases - The New Face of an Ancient Enemy as Global Public Health Emergency (2016): Brief Review and Recent Updates. Int J Prev Med 2017;8:6.

6. Pan American Health Organization / World Health Organization. WHO announces a Public Health Emergency of International Concern. February 17, 2016. Available at http://www.paho.org/hq/index.php?option=com_content\&vi ew $=$ article $\&$ id $=11640 \&$ Itemid $=135 \&$ lang $=e n$

7. World Health Organization. WHO Director- General summarizes the outcome of the Emeregency Committee regarding clusters of Microcephaly and Guilllain Barre syndrome. February 17, 2016. Available at

http://www.who.int/mediacentre/news/statements/2016/1stemergency-committee-zika/en/

8. Bennett J. India faces worst dengue fever outbreak in years with more than 6,500 confirmed cases. ABC News.

February 17, 2017.Available at

http://www.abc.net.au/news/2015-10-05/india-in-grip-ofworst-dengue-fever-outbreak-in-years/6826916

9. CDC, Division of Vector-Borne Diseases, Arboviral Diseases and Dengue Branches. Updated diagnostic testing for Zika, chikungunya, and dengue viruses in US Public. Health Laboratories. February 2, 2016.

10. http://www.cdc.gov/zika/pdfs/denvchikvzikv-testingalgorithm.pdf. Last assessed on February 2, 2016.

11. Millet JP, Montalvo T, Bueno-Marí R, Romero-Tamarit A, Prats-Uribe A, Fernández L et al. Imported Zika Virus in a European City: How to Prevent Local Transmission? Front Microbiol. 2017;8:1319.

12. Center for Disease Control and Prevention. Questions and Answers for Pediatric Healthcare Providers: Infants and Zika Virus Infection. February 2, 2016. Available at http://www.cdc.gov/zika/hc-providers/qa-pediatrician.html

13. World Health Organization. Zika Virus. January 26, 2016. Available at http://www.wpro.who.int/mediacentre/factsheets/fs_051820 15_zika/en/ 